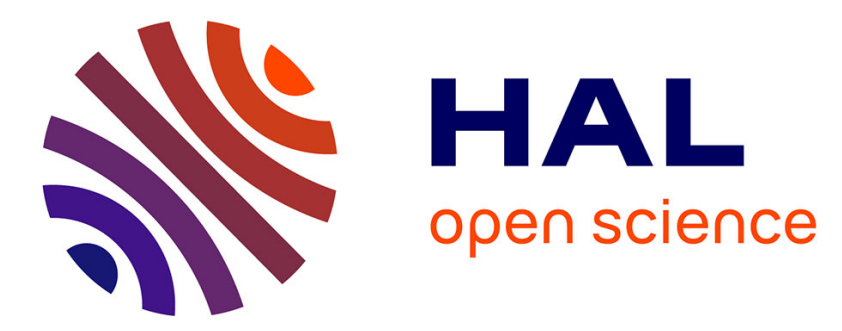

\title{
Les bibliothèques sinologiques françaises : rôles et enjeux
}

Delphine Spicq, Soline Lau-Suchet

\section{To cite this version:}

Delphine Spicq, Soline Lau-Suchet. Les bibliothèques sinologiques françaises : rôles et enjeux. Études Chinoises, 2010, Étudier et enseigner la Chine, Hors-série, p. 131-161. hal-01283816

\section{HAL Id: hal-01283816 \\ https://hal-inalco.archives-ouvertes.fr/hal-01283816}

Submitted on 6 Mar 2016

HAL is a multi-disciplinary open access archive for the deposit and dissemination of scientific research documents, whether they are published or not. The documents may come from teaching and research institutions in France or abroad, or from public or private research centers.
L'archive ouverte pluridisciplinaire HAL, est destinée au dépôt et à la diffusion de documents scientifiques de niveau recherche, publiés ou non, émanant des établissements d'enseignement et de recherche français ou étrangers, des laboratoires publics ou privés.

\section{다)(1) $\$$}

Distributed under a Creative Commons Attribution - NonCommercial| 4.0 International 


\section{Les bibliothèques sinologiques françaises : rôles et enjeux}

\section{Delphine Spicq et Soline Suchet}

L'indéniable richesse de nos bibliothèques d'études chinoises est le reflet d'une longue tradition sinologique. Dès le XVII ${ }^{\mathrm{e}}$ siècle, avant même la naissance de cette discipline, les bibliothèques françaises se sont progressivement enrichies d'ouvrages chinois, dons issus des aventures missionnaires en Chine comme des échanges proto-diplomatiques entre le royaume de France et l'empire du Milieu. À partir du XIX ${ }^{\mathrm{e}}$ siècle et de la mise en place de chaires consacrées à l'étude de la Chine, un petit nombre d'établissements prestigieux a entamé un patient travail de constitution de collections chinoises, lesquelles ont depuis lors accompagné et nourri la sinologie française.

Ce patrimoine singulier se trouve aujourd'hui confronté à des évolutions structurelles et conjoncturelles qui mettent en question les stratégies documentaires et scientifiques de nos établissements. Depuis peu, les défis lancés de manière générale au monde des bibliothèques et de l'écrit induisent de la part des tutelles ministérielles des réponses dont le caractère global risque de négliger les besoins et les contraintes propres aux bibliothèques sinologiques. Or ceux-ci sont de plus en plus exigeants : l'explosion, la numérisation et le coût croissants de la production éditoriale chinoise interrogent nos lacunes humaines, financières et technologiques, tandis que les transformations contemporaines de la recherche en général, de la recherche sinologique en particulier, poussent à réévaluer nos orientations documentaires et scientifiques.

Pour continuer de tenir un rôle central au sein de la recherche, les bibliothèques françaises d'études chinoises doivent tout à la fois réaffirmer leur spécificité au sein du paysage documentaire français et repenser leurs relations avec le monde de la recherche, en trouvant le juste milieu entre une souplesse 
adaptée aux transformations scientifiques et technologiques de celui-ci, et le maintien de leurs missions originelles.

\section{État des lieux des bibliothèques françaises de sinologie}

Des bibliothèques riches aux fonds diversifiés

\section{Tableau 1 : bibliothèques sinologiques françaises}

\begin{tabular}{|c|c|c|c|c|c|}
\hline \multirow[t]{2}{*}{ Bibliothèques } & \multirow{2}{*}{$\begin{array}{l}\text { Date } \\
\text { de } \\
\text { fondat } \\
\text { ion }\end{array}$} & \multirow[t]{2}{*}{$\begin{array}{l}\text { Type de } \\
\text { bibliothèque }\end{array}$} & \multirow[t]{2}{*}{ Spécialités } & \multirow[t]{2}{*}{ Fonds } & $\begin{array}{l}\text { Person } \\
\text { nel }^{1}\end{array}$ \\
\hline & & & & & \\
\hline $\begin{array}{l}\text { BIULO } \\
\text { (BULAC, } \\
\text { depuis le } \\
01 / 01 / 2010 \text { ) }\end{array}$ & $\begin{array}{l}\mathrm{Mi} \\
\mathrm{XVII}^{\mathrm{e}}\end{array}$ & $\begin{array}{l}\text { Bibliothèque } \\
\text { universitaire }\end{array}$ & $\begin{array}{l}\text { Littérature, } \\
\text { linguistique, histoire, } \\
\text { sciences sociales, } \\
\text { ethnologie } \\
\text { (monographies et } \\
\text { périodiques de la } \\
\text { République de Chine } \\
\text { et de Taiwan) }\end{array}$ & $\begin{array}{l}\text { Archives, monographies et } \\
\text { périodiques, cartes } \\
40000 \text { volumes }\end{array}$ & $\begin{array}{l}1+0 \\
25\end{array}$ \\
\hline BM Lyon & $\begin{array}{l}1926 \\
{[1970]}\end{array}$ & $\begin{array}{l}\text { Bibliothèque } \\
\text { publique }\end{array}$ & $\begin{array}{l}\text { Chine moderne, } \\
\text { littérature } \\
\text { contemporaine } \\
\text { histoire des idées et } \\
\text { des mouvements } \\
\text { politiques } \\
\text { Propagande, présence } \\
\text { jésuite en Chine }\end{array}$ & $\begin{array}{l}\text { Fonds Michelle Loi, } \\
\text { Jacques Guillermaz, } \\
\text { Michel Soymié } \\
\text { Collection jésuite de } \\
\text { Chantilly } \\
\text { Fonds de 1'Institut franco- } \\
\text { chinois de Lyon } \\
50000 \text { volumes }\end{array}$ & 2 \\
\hline $\begin{array}{l}\text { BnF F. } \\
\text { Mitterrand }\end{array}$ & 1996 & $\begin{array}{l}\text { Bibliothèque } \\
\text { publique }\end{array}$ & $\begin{array}{l}\text { Littérature } \\
\text { contemporaine } \\
\text { art contemporain }\end{array}$ & $\begin{array}{l}\text { Monographies et } \\
\text { périodiques } \\
26000 \text { volumes dont } 20 \\
000 \text { en chinois }\end{array}$ & 1,3 \\
\hline $\begin{array}{l}\text { BnF } \\
\text { Richelieu }\end{array}$ & 1666 & $\begin{array}{l}\text { Bibliothèque } \\
\text { publique }\end{array}$ & $\begin{array}{l}\text { Histoire, archéologie, } \\
\text { religions et } \\
\text { philosophie }\end{array}$ & $\begin{array}{l}\text { Mission Paul Pelliot } \\
\text { (manuscrits, estampages et } \\
\text { inscriptions antérieures au } \\
\text { XIe siècle, ouvrages } \\
\text { chinois), } \\
13000 \text { manuscrits, } 153500 \\
\text { titres }\end{array}$ & $2, ?$ \\
\hline $\begin{array}{l}\text { CECMC/ } \\
\text { EHESS }\end{array}$ & $1996^{2}$ & $\begin{array}{l}\text { Bibliothèque } \\
\text { de recherche }\end{array}$ & $\begin{array}{l}\text { Chine moderne et } \\
\text { contemporaine, } \\
\text { histoire et sciences } \\
\text { humaines et sociales }\end{array}$ & $\begin{array}{l}\text { Monographies et } \\
\text { périodiques } \\
20000 \text { titres chinois et } 15 \\
000 \text { titres occidentaux }\end{array}$ & 2,5 \\
\hline $\begin{array}{l}\text { CRLAO/ } \\
\text { EHESS }\end{array}$ & 1960 & $\begin{array}{l}\text { Bibliothèque } \\
\text { de recherche }\end{array}$ & Linguistique & $\begin{array}{l}\text { Monographies et } \\
\text { périodiques, } \\
16500 \text { volumes, la plupart } \\
\text { en chinois }\end{array}$ & 2 \\
\hline
\end{tabular}

\footnotetext{
${ }^{1}$ A. $=$ Acquisitions $/$ C. $=$ Catalogage et autre

${ }^{2}$ Le CECMC fait suite au Centre de documentation et de recherche sur la Chine contemporaine, fondé en 1958 par Jacques Guillermaz spécialisé dans la recherche sur la Chine du $\mathrm{XX}^{\mathrm{e}}$ siècle autour duquel fut rassemblée une importante collection de documents concernant la Chine contemporaine ainsi qu'au Centre d'études comparatives du monde Chinois créé en 1985 par Michel Cartier dont les recherches portaient sur l'histoire et la civilisation chinoises dans une perspective comparative.
} 
La communauté sinologique française dispose d'institutions de qualité, aux fonds riches et diversifiés, comptant parmi les plus anciens et les plus prestigieux d'Europe et d'Occident.

Ces fonds recèlent une véritable richesse historique, puisque leur constitution et leur accroissement sont étroitement liés à l'histoire de la sinologie française, pionnière durant plus d'un siècle, et remontent à la période pré-sinologi-

\begin{tabular}{|c|c|c|c|c|c|c|}
\hline \multirow[t]{2}{*}{ Bibliothèques } & \multirow{2}{*}{$\begin{array}{l}\text { Date de } \\
\text { fondation }\end{array}$} & \multirow{2}{*}{$\begin{array}{l}\text { Type de } \\
\text { bibliothèque }\end{array}$} & \multirow[t]{2}{*}{ Spécialités } & \multirow[t]{2}{*}{ Fonds } & \multicolumn{2}{|c|}{ Personnel $^{1}$} \\
\hline & & & & & $\mathrm{A}$ & C. \\
\hline EFEO & $\begin{array}{l}1903 \\
\text { (Hanoï) } \\
1968 \\
\text { (Paris) }\end{array}$ & $\begin{array}{l}\text { Bibliothèque } \\
\text { de recherche }\end{array}$ & $\begin{array}{l}\text { Histoire } \\
\text { ancienne, } \\
\text { archéologie, } \\
\text { religions, études } \\
\text { bouddhiques, } \\
\text { anthropologie } \\
\end{array}$ & $\begin{array}{l}\text { Monographies et } \\
\text { périodiques, estampages, } \\
\text { manuscrits anciens, } \\
\text { photographies } \\
10000 \text { titres dont } 7000 \text { en } \\
\text { chinois }\end{array}$ & 1 & \\
\hline Guimet & 1889 & $\begin{array}{l}\text { Bibliothèque } \\
\text { de musée }\end{array}$ & $\begin{array}{l}\text { Art ancien et } \\
\text { archéologie }\end{array}$ & $\begin{array}{l}\text { Archives, cartes, manuscrits } \\
\text { et estampages, rouleaux, } \\
\text { photographies et périodiques } \\
5000 \text { titres chinois }\end{array}$ & 1 & 1 \\
\hline IAO Lyon & 1992 & $\begin{array}{l}\text { Bibliothèque } \\
\text { de recherche }\end{array}$ & $\begin{array}{l}\text { Chine } \\
\text { contemporaine, } \\
\text { sciences } \\
\text { humaines }\end{array}$ & $\begin{array}{l}\text { Monographies et périodiques } \\
25000 \text { volumes }\end{array}$ & 2 & \\
\hline IHEC/CDF & 1924 & $\begin{array}{l}\text { Bibliothèque } \\
\text { de recherche }\end{array}$ & $\begin{array}{l}\text { Études classiques } \\
\text { jusqu'en } 1911\end{array}$ & $\begin{array}{l}\text { Collectanea (丵書), } \\
\text { monographies locales (地方 } \\
\text { 志), livres rares (善本) et } \\
\text { objets précieux, } \\
\text { environ } 300000 \text { volumes }\end{array}$ & 3 & 4 \\
\hline $\begin{array}{l}\text { LCAO/Paris } \\
\text { Diderot }\end{array}$ & 1970 & $\begin{array}{l}\text { Bibliothèque } \\
\text { universitaire }\end{array}$ & $\begin{array}{l}\text { Linguistique, } \\
\text { littérature et } \\
\text { civilisation }\end{array}$ & $\begin{array}{l}\text { Monographies et périodiques } \\
20000 \text { ouvrages }\end{array}$ & 1 & \\
\hline $\begin{array}{l}\text { René } \\
\text { Grousset } \\
\text { (Cernuschi) }\end{array}$ & 1954 & $\begin{array}{l}\text { Bibliothèque } \\
\text { de musée }\end{array}$ & Histoire de l'art & $\begin{array}{l}\text { Manuscrits, monographies, } \\
\text { archives, cartes, rouleaux, } \\
\text { estampages et périodiques } \\
12000 \text { titres dont } 1000 \text { en } \\
\text { chinois }\end{array}$ & 1 & \\
\hline $\begin{array}{l}\text { Société } \\
\text { asiatique }\end{array}$ & 1822 & $\begin{array}{l}\text { Bibliothèque } \\
\text { de recherche }\end{array}$ & $\begin{array}{l}\text { Histoire, } \\
\text { religions et } \\
\text { archéologie }\end{array}$ & $\begin{array}{l}\text { Nombreuses monographies } \\
\text { et périodiques } \\
\text { Fonds Chavannes, } \\
\text { Demiéville et Maspéro, } \\
21000 \text { volumes } \\
\end{array}$ & & role) \\
\hline
\end{tabular}

${ }^{1}$ A. $=$ Acquisitions $/$ C. $=$ Catalogage et autre. 
que $^{1}$. Les collections les plus anciennes remontent au XVII ${ }^{\mathrm{e}}$ siècle, à l'heure où la bibliothèque du Roi et celles des jésuites accueillent les ouvrages ramenés de Chine par les toutes premières expéditions françaises. Plus tard, la création de chaires de chinois au Collège de France (1814) puis à l'École spéciale des langues orientales vivantes (1843) amena la constitution de fonds d'étude et de recherche qui n'ont cessé de s'enrichir tout du long du XIX ${ }^{\mathrm{e}}$ siècle, et font de nos jours partie intégrante du patrimoine français. Au fil du temps, les intérêts se sont diversifiés et les collections, à l'origine davantage orientées vers les Classiques, la langue et la littérature, se sont enrichies et reflètent tout l'éventail des champs de prédilection de la sinologie française : linguistique, littérature, histoire, philosophie, archéologie, religion et, depuis une quinzaine d'années, sciences humaines et sociales.

La constitution de ces collections est très diverse. Bien qu'une grande partie des ouvrages ait été acquise sur deniers publics, les dons de particuliers ou d'institutions participent de leur richesse, puisque les fonds chinois n'ont pas été seulement constitués en amont mais également en aval de la recherche : en effet, de nombreux fonds de chercheurs, fruit de toute une vie consacrée à l'étude de la Chine, sont venus les enrichir. Le fonds de la bibliothèque du musée Cernuschi, par exemple, est constitué en majorité d'ouvrages légués par René Grousset, tandis que les collections de l'IHEC sont aussi bien le résultat des achats de bibliothécaires que de l'intégration du fonds propre de l'IHEC et de celui du Centre d'études sinologiques de l'université de Paris à Pékin. Autre exemple, le fonds chinois de la Bibliothèque municipale de Lyon Part-Dieu s'est presque entièrement constitué à partir de bibliothèques privées, depuis l'intégration de la bibliothèque et des archives de l'Institut franco-chinois de Lyon, jusqu'aux legs des collections personnelles de Michel Soymié, Michelle Loi ou Jacques Guillermaz. N'oublions pas la Société asiatique, dont le riche fonds chinois relève en grande partie de l'héritage des nombreux ouvrages de Édouard Chavannes, Paul Demiéville et Henri Maspéro. En dépit de leur richesse indéniable, les bibliothèques françaises d'études chinoises présentent un certain nombre de faiblesses structurelles et conjoncturelles.

1 Si les premières études françaises sur la Chine paraissent dès les $\mathrm{XVII}^{\mathrm{e}}$ et $\mathrm{XVIII}{ }^{\mathrm{e}}$ siècles, on ne peut encore parler de sinologie, la Chine ne faisant pas encore l'objet d'une approche voulue comme scientifique. 


\section{Un manque d'unité et de visibilité}

Ces collections relèvent en majorité d'établissements de recherche, à l'exception de deux bibliothèques publiques (BnF et BM Lyon Part-Dieu), de deux bibliothèques de musée (Guimet et Cernuschi) et de deux établissements universitaires (BULAC et LCAO université Paris-Diderot). Nous ne nous attarderons pas ici sur les quelques établissements aux collections largement occidentales, que sont le Centre Sèvres ou les Missions étrangères de Paris, non plus que sur les fonds consacrés à la Chine et relevant des sections de chinois des universités de Bordeaux 3 Michel de Montaigne, de Lyon 3 Jean Moulin et de la Rochelle, qui sont déposés dans les bibliothèques de sciences humaines de ces universités.

La diversité de statut de ces établissements, ajoutée à leur rattachement à des tutelles ministérielles ou publiques différentes, nuit à leur visibilité. C'est particulièrement le cas à l'étranger, habitué à une configuration documentaire plus homogène. Cette diversité s'accompagne d'un déséquilibre certain entre une majorité des fonds importants concentrés à Paris aux dépens de la province. Ce parisiocentrisme nuit à la visibilité et, par conséquent, à l'exploitation de fonds plus excentrés. Le meilleur exemple en est la bibliothèque municipale de Lyon Part-Dieu, dont les collections chinoises sont mal connues, notamment des jeunes chercheurs, et de ce fait sous-exploitées (cf. encadré p. 138).

Par ailleurs, les règles administratives et comptables des grands établissements publics auxquelles les bibliothèques sont soumises entraînent un manque de souplesse et compliquent souvent les processus d'acquisition des ouvrages. D'autre part, la position du bibliothécaire dans la hiérarchie interne et sa marge décisionnelle dans les stratégies budgétaire et documentaire varient grandement d'un établissement à l'autre. Ces lourdeurs administratives engendrent dans certains cas un manque d'autonomie du bibliothécaire. Pour exemple, si à l'IHEC, le conservateur est placé directement sous le titulaire de la chaire de chinois et directeur de l'institut, à la BIULO/BULAC ou à la BnF, plusieurs niveaux de hiérarchie séparent le conservateur de sa direction. Dans le cas précis des bibliothèques universitaires ou publiques, l'aspect scientifique du travail du bibliothécaire pâtit du poids croissant de politiques managériales valorisant objectifs chiffrés et productivité (en termes d'acquisition, catalogage, rotation des ouvrages, etc.) aux dépens de l'aspect scientifique (cohérence de la politique 
documentaire, qualité du catalogage, réponse aux besoins exigeant d'une communauté réduite). Une déconnection progressive entre les équipes de gestion et les équipes de bibliothécaires scientifiques amènent les premières à sous-estimer les contraintes propres à la discipline sinologique ainsi qu'à la documentation chinoise. Celles-ci sont pourtant de plus en plus lourdes et entrent parfois en conflit avec les impératifs managériaux.

Cette situation n'est pas propre à la France ; à l'université de Leiden, par exemple, la bibliothèque du département de chinois a fusionné avec les autres bibliothèques spécialisées, de sorte que les collections ont été réunies dans un seul bâtiment avec une salle de lecture commune. Le personnel de ces bibliothèques ainsi que leurs budgets sont à présent gérés par une équipe managériale, avec pour conséquence une participation de moins en moins grande des bibliothécaires aux décisions. Au final, ces derniers se voient imposer celles de leurs gestionnaires et deviennent de simples exécutants, avec parfois des résultats inquiétants, comme en témoigne la démission du bibliothécaire du département de chinois.

Pour résumer, la situation varie fortement d'un établissement à l'autre et place nos bibliothèques dans une position d'inégalité en termes d'autonomie budgétaire et scientifique. Un tel état des choses nuit à la mise en place d'une politique documentaire commune cohérente et induit une dispersion des ressources budgétaires et humaines fort dommageable.

Des tentatives de regroupement et de coopération ont été menées, sans suite. L'association SINODOC, notamment, fut lancée par Hubert Delahaye à la fin des années 1990 dans le but d'instaurer un dialogue et une coopération rapprochée. Les réunions portèrent aussi bien sur les politiques d'acquisition que sur les questions de catalogage en bi-écriture ou encore sur la constitution de catalogues collectifs spécialisés (périodiques, livres rares, etc.). Il réunissait une petite quarantaine de personnes et une vingtaine d'établissements (bibliothèques, UFR, librairies...). L'échec d'un tel projet, au début des années 2000, témoigne de la difficulté de maintenir sur le long terme une collaboration de proximité dans le cadre d'établissements aux tutelles et structures très diverses ${ }^{2}$.

2 Les comptes-rendus des rencontres de SINODOC sont en partie disponibles depuis le site Internet de l'Association européenne des bibliothécaires sinologues (EASL), à l'adresse suivante : http://easl.org/acta.html. 


\section{Diminution des moyens humains et budgétaires}

À ces facteurs structurels s'en ajoutent des conjoncturels. La politique actuelle de révision générale des politiques publiques (RGPP) entraîne depuis quelques années une baisse des moyens humains et budgétaires préjudiciable aux politiques documentaires de nos établissements. Depuis 2007, un départ à la retraite sur deux n'est plus remplacé, tandis que se dégage une tendance à la stagnation voire à la baisse des budgets. Cette dernière n'est que faiblement compensée par le recours au mécénat ou au patronage, étranger à la tradition française, à la différence des pays anglosaxons. Par ailleurs, les moyens budgétaires se restreignent à l'heure même où, sans compter le coût exponentiel des imprimés, les besoins créés par l'afflux des ressources numériques payantes appelleraient la mise en place de lignes budgétaires propres.

Ces budgets paraissent modestes au regard de nombre d'établissements étrangers, notamment anglo-saxons. Ainsi, le budget acquisitions du département des collections chinoises de la Bodleian Library d'Oxford est supérieur à 100000 euros par an ; celui du département de chinois de la Staatsbibliothek zu Berlin de plusieurs centaines de milliers d'euros alors que celui de la bibliothèque de l'IHEC, de loin le plus important en France, n'est que de 30000 euros.

Toutefois la situation varie en fonction des établissements. Il semble que les bibliothèques de recherche à aire géographique spécialisée (CECMC, EFEO, IAO et IHEC) soient relativement bien loties, en comparaison des établissements à vocation plus large (bibliothèques nationale, publiques ou universitaires), que leur mission générale empêche de consacrer leurs moyens budgétaires et humains au seul chinois. La BIULO/BULAC (17 000 euros pour les ouvrages en langue chinoise) et la BnF (budget quasi nul) font partie des cas les plus flagrants. Les budgets alloués aux acquisitions varient d'une institution à l'autre. Ils sont les plus élevés, ce qui n'est en rien surprenant, dans les bibliothèques de recherche à aire spécialisée. Encore une fois, ces chiffres restent modestes au regard des budgets de certains partenaires européens cités plus haut et il serait préjudiciable à la recherche sinologique qu'ils diminuent. 


\section{Les fonds chinois de la bibliothèque municipale de Lyon Part-Dieu ${ }^{1}$}

\section{Création}

1970, Transfert des collections de la bibliothèque de l'ancien institut francochinois de Lyon (1926-1941) au sein de la bibliothèque de la Part-Dieu (25000 ouvrages et 400 titres de périodiques)

\section{Accroissement}

Legs Jacques Guillermaz (1911-1998) de sa bibliothèque chinoise privée : 2500 volumes, livres et revues sur la période républicaine et documents sur la Révolution culturelle.

Domaines : histoire des idées et des mouvements politiques, littérature chinoise moderne, sociologie.

Don Michelle Loi (1926-2002) : 4000 documents de et sur la littérature chinoise postérieure au mouvement de mai 1919; matériaux de propagande de haute valeur documentaire

Transfert de la bibliothèque jésuite des Fontaines de Chantilly en 1999 : 12000 ouvrages spécialisés dans le domaine religieux et la présence jésuite en Chine

2003 : don Soymié (1924-2002) : 8000 volumes en langues occidentales, chinois et japonais. Reflète les acquis de la recherche sinologique au Japon.

\section{Particularité}

Les collections de la bibliothèque de l'Institut franco-chinois, à l'inverse des autres bibliothèques chinoises de France, ont été constituées par des Chinois et non par des Occidentaux.

\section{Spécialités}

Littérature moderne et contemporaine

Histoire des idées et des mouvements politiques

Religion chrétienne en Chine

\section{Volumétrie}

50000 volumes

800 titres de périodiques

25 mètres linéaires d'archives

\footnotetext{
1 Pour plus d'information, consulter le site Internet de la bibliothèque municipale de Lyon Part-Dieu qui consacre une page à son fonds chinois : http://www.bm-lyon.fr/trouver/Fonds_chinois/Fonds_chinois.htm. Cette page propose un historique détaillé des collections, une aide à la consultation du catalogue informatique, des repères bibliographiques ainsi qu'une série de liens concernant l'édition chinoise et la recherche documentaire sur Internet.
} 
En termes de moyens humains, si l'on observe la dotation en personnel de nos établissements (tableau 1), on s'aperçoit que les chiffres sont, de manière générale, assez faibles. Plusieurs bibliothèques fonctionnent avec un personnel minimal, si bien que les personnes en poste se retrouvent bibliothécaires multitâches et assument toutes les fonctions (service aux lecteurs, veille documentaire, acquisitions, réception, catalogage, désherbage ${ }^{3}$ et numérisation).

\section{Des défis}

Les bibliothèques françaises d'études chinoises sont confrontées à l'heure actuelle à des défis matériels et scientifiques qui viennent interroger, les handicaps que nous venons de présenter.

\section{L'explosion de la production éditoriale chinoise}

Depuis une vingtaine d'années, le volume et le coût de la production éditoriale chinoise ne cessent de croître ${ }^{4}$. Pour ne parler que des monographies ( $c f$. tableau 2), l'année 2008 comptait plus de 275000 parutions, dont un peu moins de 150000 nouveautés, sans compter les ouvrages scolaires, les périodiques ( $c f$. tableau 3) et les ressources numériques ( $c f$. tableau 4), ces deux dernières catégories connaissant une croissance soutenue.

3 Dans une bibliothèque le désherbage consiste à actualiser les collections en éliminant des ouvrages.

${ }^{4}$ Les chiffres et graphiques qui suivent sont issus des statistiques établies par l'Administration générale de la presse et de l'édition de la République populaire de Chine (中华人民共和国新闻出版总暑) et mises en ligne sur son site à l'adresse suivante : http://www.gapp.gov.cn 
Tableau 2 : Nombre de parutions de monographies depuis 1949

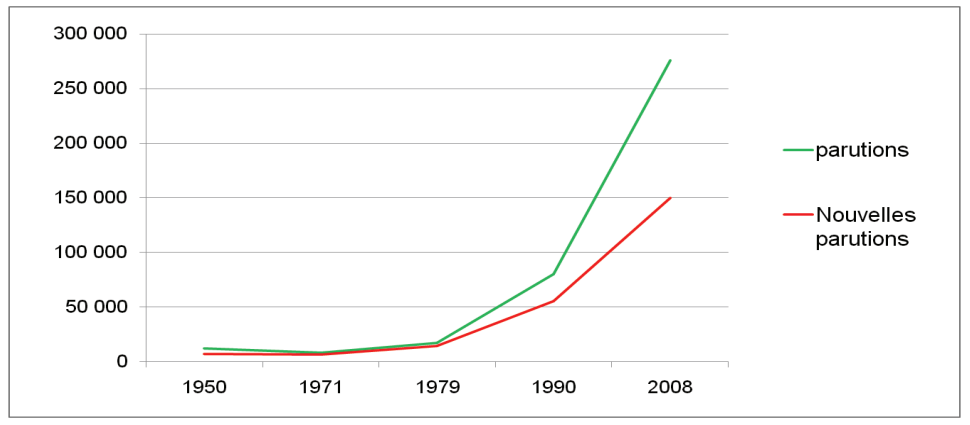

Tableau 3 : Nombre de périodiques depuis 1949

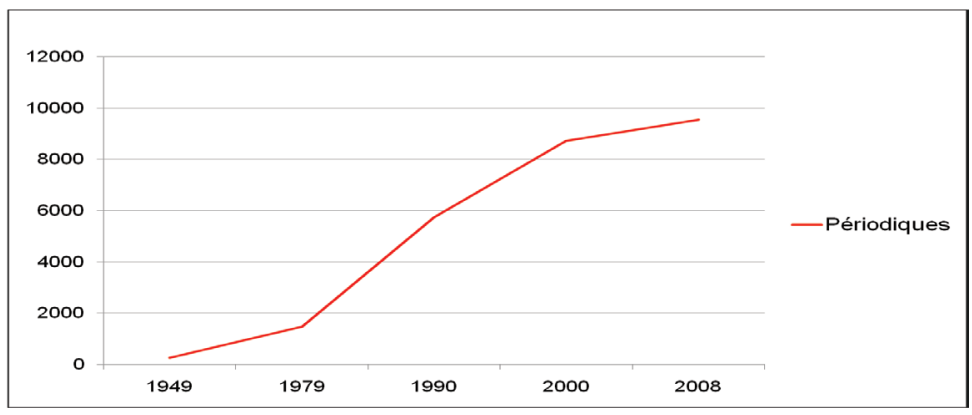

Tableau 4 : Nombre d'éditions électroniques depuis 2001

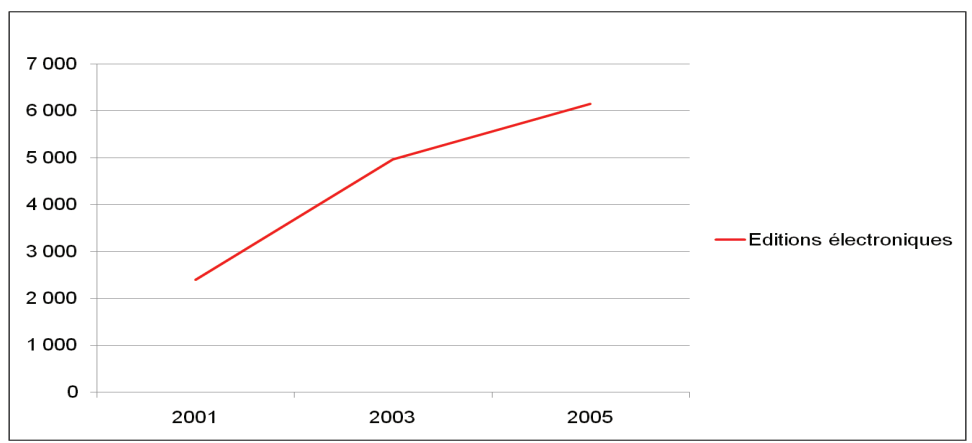


Ce phénomène s'accompagne d'une hausse des prix qui touche aussi bien les monographies ( $c f$. tableau 5), les grandes collections imprimées, si précieuses pour les bibliothèques de recherche, que les ressources numériques, de plus en plus indispensables.

\section{Tableau 5 : Augmentation du coût des monographies depuis 1996}

(en centaines de millions de RMB)

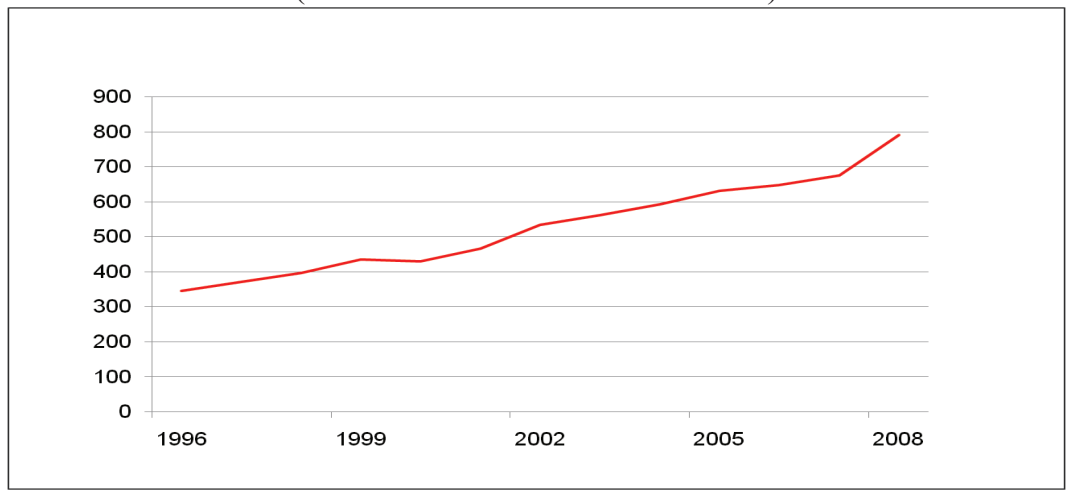

En ne considérant que la production imprimée, nos institutions sont déjà confrontées à une série de problèmes concrets. En premier lieu, en termes d'approvisionnement, un certain nombre de bibliothèques tombe sous la loi des marchés publics, qui les oblige à passer par un fournisseur unique par domaine. Ainsi est-il difficile de se fournir à la fois sur le continent, où paraissent la majorité des publications, à Hong Kong où certaines publications n'agréent pas les fournisseurs officiels, et à Taiwan, peu ou pas couvert par les fournisseurs continentaux. Ce défaut ne peut être que partiellement pallié par les dons, pourtant spontanés et généreux, de la part des bibliothèques continentales ou taiwanaises : en effet la qualité de ces derniers laisse parfois à désirer, le contenu n'étant pas exempt d'articles de propagandes ou d'invendus de faible intérêt. En second lieu, étant donné les chiffres énoncés plus haut, un suivi exhaustif des parutions est désormais impossible. Les bibliothécaires croulent sous les catalogues alors même que leurs tâches multiples les empêchent de consacrer le plus clair de leur temps aux acquisitions. En dernier lieu, les prix exorbitants imposent de faire le 


\section{Delphine Spicq et Soline Suchet}

choix souvent difficile entre une grande quantité de monographies isolées encore relativement bon marché et quelques grandes collections extrêmement chères. Or, il faut garder à l'esprit que la durée de vie de l'édition chinoise est très courte : ce qu'on n'achète pas une année risque d'être rapidement épuisé.

Bien que l'afflux des ressources numériques semble pouvoir pallier à ce dernier problème, il pose également un certain nombre de difficultés. À commencer par le coût : la cherté des ressources électroniques exigerait la mise en place de consortiums nationaux ou européens, malheureusement entravée par la complexité du paysage administratif et financier des établissements sinologiques français. Il faut par ailleurs compter avec la volatilité des prix, qui changent au gré des négociations entre les éditeurs/ fournisseurs et les établissements, d'autant que les éditeurs/fournisseurs chinois trouvent leurs principaux clients en Chine et aux États-Unis, ce qui leur permet de bouder les consortiums au profit de négociations bilatérales, ruineuses pour les établissements. Ensuite, l'incertitude afférente à la question des droits d'auteur, de consultation, de prêt et de reproduction pose un problème récurrent : ces différents droits sont encore très mal gérés en Chine et imposent une grande prudence de la part des bibliothécaires avant l'achat d'une ressource. C'est un souci particulièrement délicat dans le cas des E-books. Il suffit de donner l'exemple de Chaoxing (超星) qui a commencé par numériser en masse les ouvrages des bibliothèques (dont la Bibliothèque nationale de Chine), sans se soucier des droits d'auteurs et afférents, puis les a mis en ligne sans plus de cérémonie. Les contrats avec les ayants-droits sont progressivement négociés, mais sans aucune garantie d'exhaustivité. Autre obstacle au recours massif au numérique, l'instabilité des contenus : dans le cas d'un accès en ligne, se posent les problèmes d'accessibilité aux archives et le risque de ne pas pouvoir payer le renouvellement annuel de l'abonnement ${ }^{5}$. Enfin, il ne faut pas négliger

5 Lorsqu'un établissement s'abonne à une ressource numérique avec accès en ligne (différent de l'achat d'un cédérom), il doit généralement verser au départ une somme importante, incluant le prix de l'abonnement pour l'année en cours (variable selon le nombre d'accès sur place ou à distance désirés), qu'il faudra renouveler et renégocier chaque année. Dans certains cas seulement, l'abonnement comprend l'accès aux archives des années précédentes. Il n'est toutefois pas possible d'archiver ces données sur son propre serveur, car elles ne peuvent être consultées qu'en ligne. Si l'abonnement n'est pas renouvelé, ces documents ne sont plus consultables. 
les contraintes techniques : logiciels de lecture parfois impropres aux environnements informatiques de nos établissements, problème de l'accès à distance, accès souvent limité à un très petit nombre de postes informatiques, impossibilité - dans certains cas - de se connecter au même moment à une même ressource, l'obsolescence rapide du matériel informatique qui oblige à une mise à niveau régulière, etc.

En définitive, nous en revenons toujours à cette même question : comment choisir? Doit-on sacrifier l'imprimé au profit du numérique ? Si celui-ci pallie les difficultés d'approvisionnement et le manque d'espace de stockage, il ampute très lourdement les budgets sans certitude de pouvoir conserver ces ressources de manière pérenne. Sacrifice d'autant plus lourd que le rapport entre le prix des ressources et leur utilisation est souvent décevant, pour partie en raison de la spécialisation croissante des chercheurs, laquelle induit une faible utilisation en proportion des ressources disponibles. Ce constat ne peut qu'encourager les bibliothécaires à privilégier les ressources généralistes, comme China National Knowledge Infrastructure (CNKI) ou Wanfang data, aux dépens de ressources plus spécialisées mais pourtant d'une haute valeur documentaire, comme le Siku quanshu 四庫全書 (la bibliothèque impériale des Quatre Trésors) ou le Gujing tushu jicheng 古今圖書集成 (la grande encyclopédie compilée sous les Qing).

\section{Des besoins croissants}

Pour finir, les bibliothèques doivent faire face à des besoins croissants. Depuis une vingtaine d'années, on assiste à un engouement pour les études chinoises et à l'apparition d'un nombre de plus en plus important d'étudiants et de jeunes chercheurs, bien qu'on ait pu observer une baisse du nombre d'inscriptions ces dernières années. Parallèlement, les domaines de la recherche se spécialisent toujours davantage de fait de la multiplication des champs disciplinaires. Ces besoins de plus en plus divers et spécifiques interrogent les lacunes documentaires de nos établissements, notamment au niveau des ressources numériques. La faiblesse de ce type de ressources en France par rapport à aux situations chinoise, américaine, mais aussi européenne, est de plus en plus criante. Il suffit de se rendre sur les pages dédiées aux ressources numériques des sites web de ces bibliothèques pour s'en rendre compte. Cela place nos établissements dans une 


\section{Delphine Spicq et Soline Suchet}

position délicate entre prétention encyclopédique - à l'échelle nationale - et spécialisation croissante des champs de la recherche. Cette situation appelle, sans aucun doute, une plus grande concertation entre ces derniers.

Face à ces difficultés, quelles attitudes adopter et quelles solutions proposer? Si nos bibliothèques veulent garder un rôle central au cœur de la recherche sinologique française, il leur faut évoluer, aussi bien sur le plan des habitudes et des outils de travail que dans leurs relations avec le monde de la recherche.

\section{Replacer les bibliothèques sinologiques françaises au cour de la recherche}

Cela passe en premier lieu par un renforcement de la coopération entre enseignants-chercheurs et bibliothécaires. Celle-ci est déjà présente - de façon plus ou moins soutenue - dans certains établissements, notamment au sein des bibliothèques de recherche qui servent un centre aux programmes de recherche définis (IAO, CRLAO, EFEO, CECMC, etc.), et non pas un public élargi. Elle demande davantage d'efforts dans les autres types d'établissements, où les liens avec les chercheurs sont d'ordinaire plus lâches.

\section{Redéfinir les besoins et opérer des choix en matière d'acquisition}

Face à l'afflux des publications imprimées et numériques, les choix s'imposent et ils sont difficiles à faire. Davantage présents sur le terrain, chercheurs et étudiants de second et troisième cycle sont des mines de renseignements sur les publications récentes parues en Chine comme sur les derniers champs de spécialisation de la recherche. Aucun cadre formel ne définissant les liens entre chercheurs, étudiants et bibliothécaires, c'est sur la bonne volonté et l'initiative individuelles que repose habituellement la collaboration entre les uns et les autres. Il faut donc encourager les échanges, faire circuler les catalogues, solliciter davantage les suggestions des chercheurs, et permettre à ceux-ci d'acheter sur le terrain pour le compte des bibliothèques et sous la supervision des bibliothécaires. Toutefois le travail de sélection ne doit pas être assuré par les chercheurs et seulement en fonction des besoins de ceuxci. Le bibliothécaire doit trouver un juste milieu entre une politique de service au chercheur et les exigences d'une politique documentaire sur le long 
terme. Ceci est primordial si l'on considère les bibliothèques à vocation patrimoniale telles que l'IHEC, la BnF ou encore la BIULO/BULAC. Cette vocation appelle un enrichissement des collections en harmonie avec les fonds historiques. Les domaines de recherche vont et viennent, l'intérêt d'un instant cédant bientôt le pas à un autre champ de spécialisation. Au cœur de ce terrain changeant, les bibliothèques doivent assurer une veille documentaire qui ne sacrifie pas la cohérence de leur politique documentaire à la seule satisfaction des besoins immédiats. Il nous semble d'autre part que la constitution d'une collection cohérente doit se faire aussi bien en réponse aux besoins de la recherche qu'en amont de ceux-ci. Nous entendons par là que nos établissements doivent aussi bien répondre aux besoins que les susciter.

La collaboration entre chercheurs et bibliothécaires nous semble particulièrement cruciale dans le domaine du numérique. Ces ressources sont trop coûteuses pour que l'erreur soit permise. À titre d'exemple, la BnF y consacre un budget annuel de un million d'euros. D'où l'intérêt pour les bibliothécaires de négocier des périodes d'essai gratuit pour les faire tester par leurs usagers, puis d'observer ces usages avant d'en envisager l'achat. Cela se pratique déjà, dans une certaine mesure. Mais pour l'instant les établissements français sont particulièrement pauvres en ressources électroniques sinologiques, surtout si on les compare avec les bibliothèques d'Europe, d'Amérique ou d'Asie. Si l'on prend l'exemple du CNKI, la plupart des bibliothèques européennes de sinologie sont abonnées à l'un de ses portails au moins, alors qu'en France, seule la bibliothèque du CECMC a pu négocier deux accès au portail Chinese Academic Journals (cf. Annexe 1). 


\section{Bibliothèques sinologiques européennes ayant accès au portail Chinese Academic Journals (CNKI)}

- Katholieke Universiteit Leuven - Oost-Aziatische Bibliotheek

- Staatsbibliothek zu Berlin - Preußischer Kulturbesitz - Ostasienabteilung

- Universität München - Institut für Ostasienkunde - Bibliothek

- Technische Informationsbibliothek, Universitätsbibliothek Hannover -

Ostasienabteilung

- Universität Heidelberg - Zentrum für Ostasienwissenschaften - Sinologisches Seminar - Bibliothek

- Universität Zürich - Ostasiatisches Seminar - Chinesische Bibliothek

- Univerità degli Studi di Venezia - Dipartimento di Studi Indologici ed Estremo-Orientali - Sezione Sinologica

- Edinburgh University Library

- University of London - School of Oriental and African Studies

- Oxford University - Bodleian Library

- Bibliotekarie Stockholms universitetsbibliotek (Stockholm University Library)

- Nordisk Institut for Asienstudier (NIAS) - Bibliotek

Sources : données EASL

Une raison en est la difficulté à mettre en place un consortium. Comme nous l'avons énoncé plus haut, les fournisseurs/éditeurs chinois préfèrent négocier bilatéralement que multilatéralement. Or nos bibliothèques dépendent de tutelles administratives différentes, aux contraintes budgétaires diverses, qui pèsent lourdement sur une possible coordination, puisque tous ne pourraient pas engager les mêmes sommes. La question est toutefois à l'ordre du jour, et nos établissements n'excluent pas la possibilité d'intégrer un consortium à échelle européenne, qui aurait davantage de poids face aux fournisseurs. Un tel consortium existe déjà. Il a été mis en place sous l'égide de la Staatsbibliothek zu Berlin et regroupe plusieurs bibliothèques de sinologie européenne autour d'accès au portail Chinese Academic Journals. L'idée serait donc d'intégrer le consortium européen. 
Outre les obstacles budgétaires, il faut également rappeler l'insistance française sur l'imprimé, qui rend difficile le sacrifice d'une partie notable des acquisitions imprimées au profit du numérique. À cet obstacle psychologique s'en rajoute un autre, lié à l'apparente volatilité du numérique et que nous avons déjà évoqué : quel garantie a-t-on de son maintien dans la durée ? De sa lisibilité d'ici une vingtaine d'années ? D'où, répétons-le, la difficulté de certains choix. Concernant les périodiques, certaines bibliothèques, comme la Bodleian Library à Oxford, cessent déjà les abonnements imprimés au profit des abonnements électroniques. D’autres succombent même à la tentation du tout numérique. Les bibliothèques nord-américaines, notamment, misent de plus en plus sur les ressources électroniques. Il est d'ailleurs intéressant de noter qu'à l'atelier organisé en octobre 2009 par la Bibliothèque nationale de Taiwan, à l'intention des bibliothécaires sinologues étrangers et portant précisément sur les ressources numériques, seuls deux Européens étaient présents, $90 \%$ des participants travaillant en Amérique du Nord. Mais cette attitude n'est pas sans danger, lorsqu'on garde à l'esprit que l'accès aux ressources numériques s'accompagne rarement de la propriété de leurs archives. Toujours est-il que ces nouvelles pratiques entraînent un bouleversement des habitudes de fonctionnement et de conservation propres aux bibliothèques traditionnelles, et les établissements français devront tôt ou tard se positionner clairement vis-à-vis de ces évolutions.

\section{Améliorer la répartition et la mise en valeur de nos collections}

En premier lieu, il faut renforcer la coopération en matière d'acquisition partagée entre les différentes bibliothèques. À ce titre, il serait bon d'envisager le lancement d'un nouveau réseau national de bibliothécaires sinologues - à l'image de l'ancien SINODOC - auquel chercheurs et enseignants seraient invités à participer. Un tel réseau aurait pour objectif, entre autres, de favoriser un partage documentaire plus rigoureux, de mettre en place des initiatives collectives - journées d'études, mise en place de catalogues collectifs spécialisés, formations, etc. -, en résumé d'échanger sur les différents défis qu'affrontent nos établissements. Dans une certaine mesure, une telle démarche existe dans le réseau DocAsie, fondé à l'été 2007 et regroupant l'ensemble des bibliothèques et centres 
de documentation sur l'Asie ${ }^{6}$. Toutefois nos établissements bénéficieraient d'un réseau uniquement consacré à la Chine.

En second lieu, il devient indispensable de mettre en valeur nos ressources, au niveau national et international. Il nous paraît impératif de mieux faire connaître les fonds sinologiques français. Pour avoir participé à l'atelier organisé par la Bibliothèque nationale de Taiwan en octobre 2009, nous avons découvert une réelle méconnaissance de la part des bibliothécaires et chercheurs anglo-saxons et asiatiques des ressources françaises. Celle-ci est d'ailleurs étroitement liée à la confusion du paysage administratif de la recherche française. Pour eux, tout se trouve à la Sorbonne ou à la BnF. Les stratégies d'acquisition en ressources numériques bénéficieraient également d'une meilleure communication. L'atelier taiwanais a révélé une sous-information à l'égard des ressources électroniques développées gratuitement par les établissements chinois et taiwanais aussi bien que par les bibliothèques étrangères. Pourtant, une meilleure veille de ces projets permettrait d'éviter l'achat de ressources redondantes par rapport à ce qui existe déjà en libre accès. Ainsi, une nouvelle version électronique du Siku quanshu vient d'être mise en accès libre sur Internet Archives. Toutefois cette version scannée ne permet pas de recherche plein-texte.

D'où l'importance des rencontres nationales et internationales entre bibliothécaires, permettant de s'informer mutuellement, d'autant que ce type de rencontres devrait faciliter la formation de consortiums pour l'achat de ressources numériques en commun. Nos bibliothèques participent déjà pour la plupart à la European Association of Sinological Librarians, qui se réunie annuellement et dont les ressources numériques est l'un des sujets de prédilection ${ }^{7}$. Mais une participation plus systématique aux rencontres extra-européennes (nous pensons notamment aux associations nord-amé-

6 Le réseau DocAsie regroupe les bibliothèques de 25 institutions d'enseignement ou de recherche françaises. C'est un réseau thématique pluridisciplinaire et de compétences. Il permet de recenser les fonds spécialisés sur l'Asie, de créer ou de resserrer les liens entre les centres de documentation et de favoriser les échanges. $C f$. site Internet : http://docasie.ish-lyon.cnrs.fr/, consulté le 23.03.10. Le réseau propose tous les ans des journées d'études sur des sujets thématiques.

7 La European Association of Sinological Librarians regroupe une centaine de bibliothèques sinologiques européennes. $C f$. le site Internet de l'association : http://www.easl.org/index.html, consulté le 16.03.10. 
ricaines) élargirait probablement notre champ de vision et d'expérience. À ce titre, l'atelier taiwanais n'est que le début d'une longue série et nous espérons que d'autres collègues auront l'occasion de s'y rendre.

Pour finir, même si l'achat d'un nombre minimal de ressources numériques semble indispensable, peut-être faudrait-il repenser la mise en valeur de nos collections imprimées pour y rediriger l'attention des chercheurs. Cette question rejoint un possible débat sur l'apport du numérique à la recherche sur un plan qualitatif. L'afflux des ressources et l'apparente facilité d'accès bénéficient sûrement à une production quantitative et sans cesse renouvelée de littérature académique. Mais quantité et qualité ne sont pas forcément synonymes. Ne risque-t-on pas une certaine dispersion et une superficialité croissante de la recherche au détriment d'une bonne connaissance et exploitation de ressources imprimées déjà fort riches?

\section{Bibliothèques et Internet}

La mise en valeur de ces collections imprimées passera par une plus grande visibilité sur l'Internet. À un premier niveau, il existe des réseaux européens et nationaux recensant les ressources des bibliothèques sinologiques. Malheureusement, s'ils sont connus des bibliothécaires, ils le sont plus rarement des chercheurs : nous voulons parler de EASL et de DocAsie, déjà cités plus haut. Leurs sites recensent et décrivent brièvement les différents établissements européens (pour le premier) et français (pour le second) et proposent un lien vers leurs catalogues.

Nous pensons par ailleurs à la numérisation des collections et à la communication sur celles-ci. À ce propos, la BULAC s'attellera à la numérisation de ses collections précieuses, à commencer par ses manuscrits naxi (une vingtaine) et yi (une trentaine), tandis que l'IHEC proposera bientôt la consultation sur Internet de ses objets précieux grâce au logiciel Digitool. Ce dernier a fait ses preuves avec la mise en ligne, par la bibliothèque de Harvard, de ses collections numérisées. N'oublions pas de mentionner les efforts de la BnF pour la mise en ligne des manuscrits chinois rapportés par Pelliot sur Gallica $2^{8}$. Ce qui ne doit pas empêcher nos établissements de participer à des projets de plus grande envergure voire de

${ }^{8}$ Une importante partie du fonds Pelliot de la BnF est numérisé ou en phase de l'être. La version électronique est consultable sur le site Gallica de la $\mathrm{BnF}, c f$. http://gallica.BNF.fr/, consulté le 23.03.10. 
les solliciter, comme l'ont déjà fait la BnF et le musée Guimet dans le cadre de l'International Dunhuang Project ${ }^{9}$. Pour ce faire nos établissements bénéficieront une fois de plus de la collaboration avec le monde de la recherche. Plusieurs ressources existent déjà, fruit d'initiatives rapprochant bibliothécaires et centres de recherche (version électronique du Shuowen jiezi 說文解字 réalisée par Alain Lucas bibliothécaire au CRLAO ${ }^{10}$; les sites « Estampages chinois conservés en Europe » et « Statuettes religieuses du Henan » (en construction), réalisés avec la participation de Barbara Bonazzi, bibliothécaire à l'EFEO ${ }^{11}$, et les sites développés par l'IAO ${ }^{12}$ ).

Enfin, nous ne devons pas oublier le rôle de professionnel de l'information et de la documentation qui est le nôtre. Face à l'accès de plus en plus aisé à tous types de documents et d'informations sur l'Internet, la plus-value du bibliothécaire réside dans le référencement et la discrimination des informations, d'une part, et l'apport de contenu documentaire sur l'Internet, d'autre part. Cela passe par plusieurs médias : veille documentaire et informationnelle, suivi de l'actualité du web par la constitution de bases de signets (liste organisée de sites Internet informant sur les domaines d'intérêt de chaque établissement, $c f$. Annexes), la réalisation de guides de recherche sur l'Internet appliqués aux études chinoises ( $c f$. Annexes), voire la mise en place de bibliothèques numériques et, dans certains cas, d'environnements numériques de travail pour rapprocher la communauté des chercheurs, enseignants et étudiants ( $c f$. Annexe 2).

\section{Bibliothèques et formation des sinologues}

Plus généralement, nous avons un véritable rôle à jouer dans la formation et l'accompagnement à la recherche documentaire. Notre expérience a montré qu'étudiants et chercheurs sont souvent mal formés à l'utilisation aussi bien des ressources en bibliothèques qu'à la recherche sur Internet.

9 Cf. le site Internet http://idp.BNF.fr/, consulté le 23.03.10.

10 Jean-Louis Schott, Alain Lucas et Antoine Leblond, Shuowenjiezi, http://www. shuowenjiezi.com/, consulté le 14.03.10.

11 Jean-Pierre Drège (dir.), Estampages chinois conservés en Europe, http://www. efeo.fr/estampages/index.php, consulté le 23.03.10.

12 «Plateformes numériques de l'IAO », http://iao.ish-lyon.cnrs.fr/spip.php?rubrique47, consulté le 16.03.10. 
Quelques initiatives existent déjà : Jacqueline Nivard et Wang Ju, documentalistes au CECMC, proposent dans le cadre de l'EHESS des formations à la recherche sur Internet. On ne peut que regretter que de telles initiatives n'attirent pas un public plus fourni. Dans le cadre des bibliothèques universitaires (BULAC et LCAO) en particulier, l'intégration de véritables cours de recherche documentaire en bibliothèque et sur Internet dans le cadre des maquettes de licence et de master, serait extrêmement profitable, les étudiants/chercheurs restant souvent interdits face à la masse des ressources à leur disposition ${ }^{13}$.

Nous en revenons à l'importance de la mise en valeur de nos collections : celle-ci passe notamment par une meilleure formation de nos lecteurs aux ressources que nous leurs proposons. Car il est regrettable de constater que les budgets réduis produisent néanmoins des collections de qualité mais souvent sous-exploitées. Bien plus, comment porter un regard réflexif et pertinent sur la sinologie française si l'on méconnaît les établissements qui l'ont nourrie et dont les collections demeurent le témoignage tangible des intérêts et de la production scientifiques de générations de sinologues français?

\section{La BULAC : un nouvel acteur?}

Le paysage que nous venons de décrire est en perpétuelle évolution. Depuis le $1^{\text {er }}$ janvier 2010, la Bibliothèque interuniversitaire des langues orientales (BIULO) a fusionné avec le GIP BULAC, dont la mission est d'ouvrir en septembre 2011 un nouvel établissement, la Bibliothèque universitaire des langues et civilisations (BULAC), réunissant un certain nombre de bibliothèques et de centres de documentation spécialisés, comme l'indique son nom, dans les langues et civilisations et qui sera installée rue des Grands Moulins, dans le $13^{\mathrm{e}}$ arrondissement de Paris, dans un bâtiment qui accueillera également l'INALCO. L'on est en droit de se demander dans quelle mesure ce que nous appellerons ce « faux » nouveau partenaire saura répondre, dans le domaine sinologique, aux défis décrits plus haut.

$13 C f$. Gilles Guiheux, "Les matériaux pour la monographie d'un territoire chinois. Le cas d'une ville marchande ", terrains \& travaux, 2009-16, p. 153174. 


\section{Un faux nouveau partenaire}

Si nous parlons de « faux » nouveau partenaire, c'est que si l'on s'en tient aux ressources sinologiques, la BULAC reprendra pour l'essentiel les fonds de la BIULO.

Rappelons que l'une des ambitions du projet du Pôle des langues et civilisations, pôle d'enseignement et de recherche dans lequel s'inscrit la BULAC, était de regrouper en un même lieu et une même structure lieux d'enseignement et fonds documentaires dispersés et spécialisés dans les langues et civilisations. À l'inverse d'autres collections (notamment russes et persanes), les collections chinoises ne bénéficieront que dans une faible mesure de ce projet puisque les bibliothèques de l'EFEO et du CECMC, principaux partenaires sinologiques, ne viendront pas rue des Grands Moulins. Bien plus, l'annonce du projet Condorcet, à Aubervilliers, qui devrait réunir une partie des établissements et centres de documentation de l'EHESS, fait entrevoir une dispersion géographique au final plus importante qu'auparavant. Concernant l'EFEO et le CECMC, un accord serait passé selon lequel lesdites bibliothèques garderont les fonds dits « vivants », c' est-à-dire servant aux besoins immédiats des chercheurs des deux centres, le reste étant mis en dépôt à la BULAC. Le partage des périodiques et des monographies entre l'EFEO et la BULAC a d'ailleurs déjà eu lieu. Aucune décision formelle, toutefois, n'a été prise quant à l'avenir de la bibliothèque du $\mathrm{CECMC}$, et ces incertitudes pèsent sur la définition d'une politique documentaire cohérente, toujours dans ce souci exposé plus haut de répartition stratégique des politiques d'acquisition entre les divers établissements spécialisés dans les études chinoises. Toutefois, ces établissements continueront de participer au périmètre BULAC, dont ils partagent le catalogue et certaines ressources numériques (Siku quanshu, Japan Knowledge, TBRC - Tibetan Buddhist Research Center -, etc.). Il n'est pas impossible d'espérer voir la mise en place de consortium dans le cadre de ce périmètre.

\section{Les futurs fonds sinologiques de la BULAC}

À l'heure actuelle, il est donc malaisé d'exposer précisément à quoi ressembleront les fonds chinois. Ce que nous pouvons affirmer d'ores et déjà, c'est que la double ambition de la BULAC de servir à la fois un public étudiant de premier cycle et un public de master, doctorants et enseignants-chercheurs, 
la place dans une position délicate, entre bibliothèque universitaire - tournée vers l'apprentissage - et bibliothèque de recherche spécialisée. Étant données les contraintes humaines et budgétaires déjà énoncées, il ne saura, bien sûr, être question d'offrir une bibliothèque de pointe dans la totalité des champs disciplinaires. Dans la perspective du retrait du CECMC et de l'EFEO, les fonds chinois s'articuleront autour de trois priorités :

- un fonds spécialisé d'apprentissage de la langue chinoise et des diverses langues et dialectes du monde chinois

- une bibliothèque de références dans les différentes disciplines, sciences dures mises à part

- un fonds de recherche dans les domaines d'excellence traditionnels de l'ancienne Bibliothèque interuniversitaire des langues et civilisations orientales (littérature, histoire, apprentissage et enseignement de la langue chinoise) et dans certains domaines développés depuis quelques années, dont les sciences sociales. Parmi ces dernières, une attention particulière sera portée à la sociologie. On cherchera enfin à développer des fonds de qualité sur les minorités de Chine (histoire et société, l'aspect religieux étant couvert par l'EFEO et l'aspect linguistique par le CRLAO), la géographie chinoise, et pour finir, la littérature et la civilisation taiwanaises.

Ces grandes lignes n'excluent pas, bien au contraire, une réponse aux besoins ponctuels et spécialisés des chercheurs et étudiants de l'INALCO. L'intérêt de la BULAC réside en effet dans la seconde grande ambition du Pôle des langues et civilisations, à savoir le rapprochement des lieux d'enseignement et de recherche avec les fonds documentaires. Cette proximité offrira une opportunité à saisir, les fonds documentaires traditionnellement rattachés à l'INALCO n'ayant que trop longtemps souffert de la dispersion des centres d'enseignement (six lieux d'enseignement) et de documentation (trois bibliothèques, sans compter les fonds délocalisés au CTL ${ }^{14}$ et à Malakoff).

\section{Quelques données concrètes}

La BULAC ouvrira en septembre 2011 rue des Grands Moulins, dans le 13 ${ }^{\text {e }}$ arrondissement de Paris et sera rejointe par l'INALCO à la rentrée de l'année universitaire 2011/2012. La volonté d'entretenir des liens plus étroits

${ }^{14}$ Le centre de linguistique appliquée (Centrum Toegepaste Linguïstiek) de l’université Hasselt en Belgique. 


\section{Delphine Spicq et Soline Suchet}

avec les étudiants et les enseignants-chercheurs se manifestera dans l'espace par l'offre d'un libre-accès réparti sur deux niveaux - étude et recherche - sans que le passage de l'un à l'autre soit imperméable (les usagers du niveau étude pourront consulter et emprunter les documents du niveau recherche et vice versa).

Les salles de lecture de l'espace étude accueilleront les étudiants de l'INALCO dont les enseignements auront lieu dans le même bâtiment. Mais elles seront également ouvertes à l'ensemble des étudiants d'Île-de-France ou des autres régions qui auront usage de ses collections. 550 places câblées, réparties dans les espaces de lecture et dans de petites salles de travail de groupe, leur seront dédiées. L'objectif est de proposer à ce niveau un ensemble de 50000 ouvrages, choisis pour accompagner les premières années d'études universitaires. Pour le chinois, l'objectif est de proposer à la rentrée 2011 un libre-accès de 4875 ouvrages, à $80 \%$ en langue occidentale et $20 \%$ en langue chinoise, et à terme, un ensemble de 6500 ouvrages. Les bibliothécaires participeront, au-delà de la fourniture de documents, à la formation des étudiants. Des visites et formations à l'usage des ressources imprimés et numériques de la bibliothèque seront régulièrement proposées, mais il faudra également envisager la mise en place de véritable cours de méthodologie documentaire et l'insistance sur l'aide ponctuelle à la recherche, à l'image des bibliographers ou reference librarians anglo-saxons.

La BULAC espère par ailleurs créer des liens étroits avec les seconds et troisièmes cycles, ainsi qu'avec l'ensemble des chercheurs. À commencer par une collaboration dans le domaine des acquisitions : l'achat par les chercheurs amenés à se rendre régulièrement en Chine, à Taiwan, à Hong Kong et autres lieux de leurs terrains de recherche, sera encouragé, dans le cadre de missions préalablement réfléchies de conserve avec les bibliothécaires. L'expérience ayant montré l'insuffisante formation des étudiants de master, doctorants et chercheurs à la recherche documentaire, il est prévu de mettre en place des formations spécifiques, appliquées aux domaines disciplinaires des uns et des autre. On travaillera également en commun à la réalisation de contenus documentaires (bibliographies, projets de numérisation, guides de recherche, etc.). Cette collaboration prendra enfin la forme de services spécifiques pour faciliter l'usage de la bibliothèque : enseignants et chercheurs pourront, par exemple, réserver des bureaux ou des salles et y entreposer des ouvrages afin de favoriser le travail en groupe des équipes de recher- 
che ou les enseignements basés sur l'utilisation de documents originaux. Par ailleurs, une partie de ces espaces sera accessible à des horaires élargis. Concernant la masse documentaire, la BULAC proposera au niveau recherche 360 places et plus de 200000 documents. Pour la Chine, 8500 ouvrages sont attendus en libre accès pour la rentrée 2011, environ 11000 à terme. Il s'agira principalement de sources primaires et d'études spécialisées, à $75 \%$ en langue originale, $25 \%$ en langue occidentale.

Espérons que ce rapide état des lieux attirera l'attention des sinologues sur la richesse de nos établissements, ainsi que sur la nécessité de resserrer les liens et la collaboration entre bibliothécaires et chercheurs. Celle-ci devrait être d'autant plus aisée que les premiers sont en grande majorité issus du monde de la recherche. Toutefois, étant donné l'éventail croissant des domaines d'intérêt de la sinologie, il est indispensable de combiner l'expertise des uns et des autres : de même qu'aucun chercheur ne peut prétendre dominer l'ensemble des disciplines, chaque bibliothécaire a sa propre spécialité, forcément limitée. Mais à se poser la question de la «sinologie introuvable », il semble qu'une partie de la réponse soit à chercher au sein du paysage documentaire français. Depuis deux siècles déjà, celui-ci s'efforce non seulement d'accompagner les élans de la sinologie, mais aussi de les inspirer. Pour ce faire, nos établissements doivent redéfinir leurs priorités et, sans pour autant abandonner leur mission patrimoniale - pour les bibliothèques concernées -, apprendre à s'affranchir d'une vision pour l'instant encore trop tournée vers l'imprimé et la simple fourniture de documents. Pour maintenir leur rôle au cœur de la recherche, il leur faut parvenir, dans la mesure du possible, à définir en commun une politique documentaire qui réponde aux évolutions de la sinologie, tout en apprenant à occuper l'espace de créativité et d'avenir élaboré par Internet. C'est là, entre autre, que le rôle de professionnel de l'information prend une toute nouvelle ampleur, face à des étudiants et des chercheurs parfois dépassés par la masse des ressources à leur disposition. 


\section{Annexe 1}

\section{RESSOURCES NUMERIQUES DISPONIBLES EN BIBLIOTHEQUE}

\section{Ressources proprement sinologiques/en chinois}

\section{- Base de données bibliographiques}

- Bibliographie of Asian Studies

(accès : Maison de 1'Asie, bibliothèque du LCAO). Elle donne accès aux références d'articles parus dans environ 500 revues, dans tous les domaines des études asiatiques, depuis 1971 (pas de texte intégral mais des références bibliographiques). L'état de collection de chaque revue est indiqué dans la liste alphabétique « Browse the BAS by Journal Title ». La plupart des revues concernées sont publiées en langues occidentales. La base référence également des chapitres d'ouvrages, des actes de colloque, des anthologies, des mélanges consacrés à l'Asie. Accès depuis les postes en salle, ou à distance sur identifiant et mot de passe.

\section{- Bases de données d'articles en texte intégral}

- CNKI (accès : Maison de l'Asie ; après-midi).

Base de données de revues académiques offrant l'accès au texte intégral des articles. Seule le poste public de la salle de lecture de la Maison de l'Asie y donne accès. L'abonnement du CECMC a été négocié pour les années 1994 jusqu'à maintenant - à l'exception des derniers numéros, progressivement intégrés, - et pour les tranches $\mathrm{F}$ « littérature et histoire », $\mathrm{G}$ « Politique/Affaires militaires/Loi », $\mathrm{H}$ « Education/Sciences sociales » et $\mathrm{J}$ « Economie/Management».

-人大复印资料 (accès : Maison de l'Asie ; après-midi).

Depuis une dizaine d'année, le centre des sciences sociales de l'université Renmin 人民大学 numérise des séries entières d'articles de revues et journaux spécialisés en sciences humaines et sociales, compilées par le centre et publiées sous forme imprimée depuis 1958. Pour l'instant, seules les séries depuis 1995 jusqu'à 2006 ont été numérisées. Cela correspond à plus de 3000 journaux et revues spécialisées en sciences humaines et sociales. 


\section{- Bases de données spécialisées}

- 四庫全書 (accès : Maison de l'Asie et rue de Lille).

Le Siku quanshu est également disponible sous forme imprimée à la bibliothèque de l'IHEC. Par ailleurs, le site "Internet Arches" donne accès gratuitement en texte intégral au Siku quanshu, suite à un projet de numérisation mis en place par les bibliothèques sinologiques américaines. Par contre le site ne bénéficie pas des modalités de recherches très pratiques de l'édition payante (notamment la recherche plein-texte). Voir : http:// www.archive.org (Recherche en caractères traditionnels : 四庫全書 ou 四 庫全書會要)

-人民日报 (accès : Maison de l'Asie).

CHANT (accès : EFEO contacter le webmaster : webmestre@efeo.fr).

\section{Ressources généralistes en langue occidentale}

\section{- Bases de données bibliographiques}

- Francis (accès : disponible à la Maison de l'Asie et au LCAO). Base de données de recherche d'articles et d'actes de congrès dans le domaine des sciences humaines et sociales.

- Bases de données d'articles en texte intégral

- JSTOR (accès : sur place à la Maison de l'Asie et à la bibliothèque du LCAO, à distance pour les étudiants de l'EHESS et du LCAO, avec login et mot de passe).

La liste des 22 revues sur l'Asie, accessibles via JSTOR, est disponible sur le guide des ressources sinologiques en ligne réalisé par Jacqueline Nivard, du CECMC. http://chineressourceselectroniques.wordpress.com/apropos/

- Project Muse (accès : sur place à la Maison de l'Asie et à la bibliothèque du LCAO, à distance pour les étudiants de l'EHESS et du LCAO, avec login et mot de passe).

La liste des 24 revues sur l'Asie accessibles via Muse est disponible sur le guide des ressources sinologiques en ligne mentionné au point précédent. Le Project Muse donne principalement accès aux archives de la fin des 
années 1990 et des années 2000, et non à celles plus anciennes.

- Cairn est accessible sur place à la bibliothèque municipale de Lyon, la Maison de l'Asie et l'IAO, et à distance pour les membres de l'IAO et de l'EHESS, avec identifiant et mot de passe.

- Sage online-journals (accès : sur place à la Maison de l'Asie et à distance pour les étudiants de l'EHESS, avec identifiant et login).

Accès à plus de 560 revues spécialisés dans le commerce, les sciences humaines et sociales, les sciences et la médecine.

\section{- Bases de données spécialisées}

- Factiva (accès : Maison de l'Asie, BULAC).

Base de données de presse proposant de nombreux titres chinois.

- BBC monitoring 's online video (accès : sur place à la Maison de l'Asie). Cette ressource couvre l'actualité de plus de 150 pays, à partir d'un large éventail de sources : radio, télévision, presse écrite, dépêches d'agences de presse, etc. Ce service offre la possibilité de rechercher dans les archives (jusqu'en 1997, mais la base de données intègrera au fur et à mesure les archives antérieures à cette date).

- China News Analysis (accès : sur place à la Maison de l'Asie).

Cette ressource propose la version numérisée de la newsletter fondée en 1953 à Hong Kong par le Père Laszlo Ladany. Elle inclut les 1625 numéros et les index annuels. 


\title{
Annexe 2 \\ DEUX EXEMPLES DE GUIDES DE RECHERCHE SUR INTERNET APPLIQUÉS AUX ÉTUDES CHINOISES
}

\author{
Chaziliao 查资料 \\ http://chaziliao.org
}

Réalisé par Pascale Coulette, bibliothécaire au Centre d'études de l'Asie de l'Est (Cétase) de l'université de Montréal, ce guide des études chinoises sur Internet propose :

- une rubrique Généralités de la discipline : encyclopédies et dictionnaires, manuels introductifs, dictionnaires de langue, guides bibliographiques, bases de données, revues académiques, bibliothèques et centres spécialisés,- une rubrique Langue chinoise : sites et manuels généraux, phonétique et transcription, écriture et calligraphie, grammaire, compréhension orale, compréhension écrite, langue classique,- une rubrique Monde chinois : profils régionaux, liens officiels, média et actualités,- une rubrique - extrêmement utile - Où trouver : articles, biographies, blogues, cartes et atlas, chronologies, classiques, contenus audio et vidéo, images, livres, romans, sites web, statistiques, thèses, traductions,- des capsules d'information : sinologie et numérisation, chinois et informatique, Internet chinois, étudier en Chine.

Ce guide est une ressource particulièrement utile pour les jeunes chercheurs et permet de s'orienter rapidement sur l'Internet.

- Ressources électroniques http://chineressourceselectroniques.wordpress.com/

Ce site, réalisé par Jacqueline Nivard, ingénieur d'études à l'EHESS, est le fruit des formations à la recherche documentaire appliquée à la sinologie, organisées par Jacqueline Nivard et Wang Ju, également ingénieur d'études à l'EHESS. Il propose des astuces de recherche sur Internet, notamment pour la recherche d'articles. Il offre par ailleurs d'utiles conseils pour la constitution de bibliographies ou de feuilles de style. 


\section{Annexe 3}

\section{EXEMPLES DE REPERTOIRES DE RESSOURCES}

Plusieurs établissements proposent sur leur site des répertoires de ressources, listes organisées de sites Internet intéressant les domaines de prédilection de chaque établissement et permettant d'orienter rapidement les recherches sur internet, en fonction de ces domaines de spécialisation.

La plupart des répertoires énumérés ci-dessous sont réalisés sur des « netvibes » ou des « delicious », sites agrégateurs de lien. L'avantage de « Netvibes » par rapport à « delicious » est de permettre l'abonnement aux flux RSS des sites enregistrés, offrant ainsi accès à une mise à jour automatique des dernières informations publiées sur ces sites.

\section{- Signets des bibliothèques de la Maison de l'Asie}

http://www.netvibes.com/maisondelasie\#Catalogues_et_moteurs_de_ recherche

Ces signets ont été établis par Barbara Bonazzi, bibliothécaire de l'EFEO, pour l'ensemble des bibliothèques hébergées à la Maison de 1'Asie, à savoir les bibliothèques des centres suivants : CECMC (EHESS), Archipel (Groupe d'études insulindiennes, EHESS), CDAT (Centre de documentation sur l'aire tibétaine, EPHE), CEIAS (Centre d'études sur l'Inde et l'Asie du Sud, EHESS), CRC (Centre de recherches sur la Corée, EHESS), CRLAO (Centre de recherches linguistiques sur l'Asie orientale, EHESS), EFEO (École Française d'Extrême Orient).

\section{- Liens de l'Institut d'Asie orientale (IAO)}

Les liens de l'IAO se partagent entre liens utiles à la recherche et liens pour une première orientation, correspondant davantage à un niveau étude.

Les liens « recherche » distinguent les sites en langues occidentales et en langues orientales. 
http://www.netvibes.com/fannyhenriot\#General_\%26_Outils_de_ reference

Les liens d'orientation se concentrent sur les sites généraux consacrés à l'Asie, les centres et associations d'études asiatiques, les institutions internationales et les fondations de soutien aux études asiatiques.

http://www.netvibes.com/signetiaog\#Sites_generaux_ sur_1\%E2\%80\%99Asie

\section{- « Netvibes » du Centre Chine}

http://www.netvibes.com/chinedoc\#Actualites_du_CECMC (netvibes) Ce «netvibes » présente les actualités du Centre, ainsi que les principales ressources en ligne francophones sur la Chine (blogs, revues, textes numériques).

\section{- « Delicious » de la BULAC}

Ce « delicious » propose des sites Internet intéressant l'étude de l'ensemble des langues et civilisations couvertes par la BULAC. Ces sites sont indexés par des «tags » (mots-clés) géographiques et thématiques, permettant une recherche croisée. Pour limiter sa requête au domaine chinois, cliquer sur le « tag » CHINE. Accès direct aux liens sur la Chine : http:// delicious.com/BULACPRO/CHINE.

Ce « delicious » est en cours d'élaboration et donc loin d'être abouti. Il est disponible depuis l'url suivante : http://delicious.com/lemondensignets/, ou bien depuis le nouveau site de la BULAC (http://www.bulac.fr/), sous la sous rubrique « Le monde en signets » (http://www.bulac.fr/la-bulacaujourdhui/les-collections/le-monde-en-signets/) de la rubrique « Collections $»$. 